\title{
Exercise stress testing: Time to restart in nuclear cardiology?
}

On January 9, 2020, a novel coronavirus (Severe Acute Respiratory Syndrome - Coronavirus 2, SARS-COV-2) was reported as the causative agent of a cluster of pneumonia cases in Wuhan, Hubei Province, China. ${ }^{1}$ Over the next few months, SARS-COV-2 spread worldwide, causing the Coronavirus Disease 2019 (COVID-19) pandemic. During this period, we have followed in our laboratory the national and international recommendations for COVID-19 prevention and control. Nuclear cardiology studies were scheduled on specific days, using one-day and (when indicated) stressonly protocols. Due to the high risk of droplet production, exercise stress testing was discouraged over pharmacological stress, and particularly regadenoson. ${ }^{2}$ Previous studies have shown that there is no significant difference between stress modalities regarding the diagnostic performance of myocardial perfusion imaging (MPI). ${ }^{3}$ However, exercise testing remains the first choice for performing the stress component of MPI study, given the significant diagnostic and prognostic information obtained, as well as the better image quality (lower gastrointestinal activity and higher target-background ratio). ${ }^{3}$ As the COVID-19 infection recedes in Europe, we have managed to increase the number of nuclear cardiology studies performed. In some cases, we have also conducted exercise stress testing combined with the use of face masks for the prevention of droplet transmission, a practice that was well-tolerated by the patients.

After weeks of decline in COVID-19 transmission rate in several European countries, we believe that it is time to restart performing exercise stress testing, either in nuclear cardiology studies or as an independent tool in cardiology patients. On the other hand, stress echocardiography, as an alternative to exercise stress testing (although of significant higher cost), involves close contact between the patient and the performing physician, which also poses a threat for COVID-19 transmission. Therefore, the choice of stress testing should be adapted considering the epidemic status, patient COVID-19 risk of transmission, and the local recommendations.

$$
\begin{array}{r}
\text { G. Angelidis, }{ }^{a} \text { V. Valotassiou, }{ }^{a} \text { D. Psimadas, }{ }^{a} \\
\text { P. Georgoulias }{ }^{a} \\
{ }^{a} \text { Nuclear Medicine Laboratory, University of Thessaly, Larissa, }
\end{array}
$$
Greece

\section{Disclosures}

AG. Angelidis, V. Valotassiou, D. Psimadas, and P. Georgoulias declare no conflict of interest.

\section{References}

1. MacIntyre R, Wang Q. Physical distancing, face masks, and eye protection for prevention of COVID-19. Lancet 2020;S01406736(20):31183-1.

2. Skali H, Murthy VL, Al-Mallah MH, et al. Guidance and best practices for nuclear cardiology laboratories during the coronavirus disease 2019 (COVID-19) pandemic: An information statement from ASNC and SNMMI. J Nucl Cardiol 2019;2020:1-8.

3. Verberne HJ, Acampa W, Anagnostopoulos C, et al. EANM Procedural guidelines for radionuclide myocardial perfusion imaging with SPECT and SPECT/CT: 2015 Revision. Eur J Nucl Med Mol Imaging 2015;42(12):1929-40.

doi:10.1007/s12350-020-02234-w

Publisher's Note Springer Nature remains neutral with regard to jurisdictional claims in published maps and institutional affiliations. 